\title{
Landau-Ginzburg method applied to finite fermion systems: Pairing in Nuclei
}

\author{
M. K. G. Kruse ${ }^{1}$, H. G. Miller ${ }^{1}$, A. R. Plastino ${ }^{1}$, \\ A. Plastino ${ }^{1,2}$ and S. Fujita ${ }^{3}$ \\ ${ }^{1}$ Department of Physics, University of Pretoria,Pretoria 0002, South Africa \\ ${ }^{2}$ La Plata Physics Institute, National University La Plata and CONICET \\ C. C. 727, 1900 La Plata, Argentina \\ ${ }^{3}$ Department of Physics, SUNY at Buffalo, Buffalo, New York, USA
}

\begin{abstract}
Given the spectrum of a Hamiltonian, a methodology is developed which employs the LandauGinsburg method for characterizing phase transitions in infinite systems to identify phase transition remnants in finite fermion systems. As a first application of our appproach we discuss pairing in finite nuclei.
\end{abstract}

PACS: 21.10.Ma, 21.10.Re, 74.20.Rp, 74.20.De 
Recently it has been pointed out that empirical evidence exists for a pairing phase transition to occur in symmetric nuclear matter at normal nuclear densities at $T_{c} \approx 8 \mathrm{Mev}$ [1, 2]. Here the energy density and the specific heat have been obtained from a finte temperature extension of the semi-empirical mass formula [3]. A Landau Ginzburg treatment of this transition together with a simple pairing calculation strongly suggests that its origin is due to the existence of a paired superconducting phase at temperatures below $8 \mathrm{Mev}[4]$. This result is not surprising as finite temperature BCS calculations in nuclear and neutron matter have suggested that such a phase should exist [5, 6, 7, 8, 9, 10, 11, 12, 13, 14, 15, 16].

One of the intriguing questions that arises is whether or not a remnant of this phase transition survives in finite nuclei. Clearly a universal feature of finite nuclei is a significant change in the density of states at excitation energies of $10 \mathrm{MeV}$ or less [17]. At lower excitation energies the spectrum of most nuclei is sparse and dominated by a relatively small number of collective states. With increasing excitation energy, the independent particle degrees of freedom dominate and the density of states grows exponentially. As the mass number increases, the low-lying collective portion of the energy spectrum becomes more compressed and an abrupt change in the many particle density of states occurs at lower excitation energies. It has, therefore, been suggested that a collective to non-collective phase transition occurs in finite nuclei [18]. Strictly speaking it is incorrect to speak of phase transitions in finite systems. No one can deny, however, that transitions between different regimes do take place in these systems, and that they are more or less abrupt. (A similar situation is known in biophysics. The helix-coil transition in certain biological molecules in solution occurs with the temperature width of around 5 degrees.) This issue, particularly in deformed systems, has been clouded by the fact that finite temperature mean field calculations have suggested that this phase transition is simply due to a drastic change of shape 19]. The deformed-to-spherical shape transition seen in these calculations is not seen in exact canonical calculations [20, 21, 22, 23] and may be an artifact of the finite temperature mean field approximation and also depends on the volume of the system [24, 25]. In spite of the fact that the canonical partition function above the critical temperature is dominated by the single particle degrees of freedom a few collective states still contribute and are extremely important in calculation of shape dependent parameters. Recent calculations of the ensemble average of the quadrupole moment squared $Q^{[2]} \cdot Q^{[2]}$ indicate that it is discontinuous in the finite temperature mean field approximation, while no discontinuity is observed 
in the canonical calculations [26]. In both cases this quantity does not appear to vanish at the critical temperature. It should also be noted, however, that when thermal fluctuations in the shape dependent order parameters are taken into account, either by macroscopic or microscopic procedures, reasonable agreement with the exact canonical calculations [20] is obtained. With increasing temperature, however, it is expected that these collective degrees of freedom will eventually completely dissolve, presumably below the critical temperature of the liquid-to-gas phase transition.

However, it should be noted that model studies in a $\mathrm{SU}(2) \times \mathrm{SU}(2)$ system show that, in the thermodynamic limit, this system exhibits a singularity in the specific heat characteristic of a true phase transition [27, 28]. Furthermore, the remnant of this singularity remains in the form of a peak in finite sytems of this type. The presence of this peak has been used to map out the phase structure in such a model [29].

In spite of the fact that in many microscopic variational calculations (see for example [30]) pairing transitions appear to take place they are difficult to identify in exact shell model calculations [31]. We propose in the present work to identify the existence of a pairing transition empirically in finite nuclei in the following manner. In the Canonical Ensemble the partition function for a nucleus of mass $\mathrm{A}$ is given by

$$
\mathbf{Z}(A, T)=\sum_{i}^{n} g_{i} \exp \left(-\beta E_{i}\right)+\int_{E_{n}}^{E_{\max }} d E g_{A, Z}(E) \exp (-\beta E)
$$

where $\beta=\frac{1}{T}, g_{i}=2 j_{i}+1$ is the spin degeneracy factor, $E_{i}$ the energy of the $i$ th state of the nucleus and $g_{A, Z}(E)$ its level density with

$$
g_{A, Z}(E)=\frac{\sqrt{\pi}}{12} \frac{\exp (2 \sqrt{a U})}{a^{\frac{1}{4}}} U^{\frac{5}{4}}
$$

where $U=E-P(Z)-P(N)$ and $a=A[0.00917 S+0.142]$ per MeV for undeformed nuclei, or $a=A[0.00917 S+0.120]$ per $\mathrm{MeV}$ for deformed nuclei. $A$ is the mass number and $S$ is the spin energy. Given experiment information about the bound states of a nucleus and an experimental fit to its continuum level density, the partition function of a nucleus can be determined empirically

From $\mathbf{Z}$ it is easy to determine as a function of $\mathrm{T}$ the excitation energy

$$
\mathrm{E}=-\frac{\partial}{\partial \beta} \ln \mathbf{Z}
$$

and the specific heat 


$$
\mathrm{C}_{\mathrm{v}}=\frac{\partial \mathrm{E}}{\partial T}
$$

A second order phase transition such as a pairing phase transition in an infinite system should lead to a discontinuity in $\mathrm{C}_{V}$ at $T_{c}$. Clearly such behavior is not posible in finite sytems. Only a remnant remains in the form of peaked structure in the specific heat. In order to ascertain whether this structure is consistent with a pairing phase transition we make use of Landau-Ginzburg theory to demonstrate that a description of the condensed or paired phase close to the critical temperature, $T_{c}$, from information in the normal or uncondensed phase obtained from experimental data can be can be accomplished. The magnitude of the remnant of this discontinuity in $C_{V}$ is compared with a simple analytical calculation for a pairing phase transition [4] corrected for finite size effects [32].

Landau and Ginzburg have provided a simple theory of phase transitions which approximates the free energy in the region around $T_{c}$ and is most useful in analyzing the thermodynamics in this region. In particular, using only knowledge about the uncondensed phase one is able to make predictions about quantities in the condensed phase,such as specific heat, magnetic susceptibility and compressability. Moreover, Landau-Ginzburg theory can be derived from microscopic considerations [4].

In the Landau Ginzburg formulation it is necessary first to determine an expression for the free energy $F(T)$ in both phases. In the following the subscript 1 will refer to the lower temperature (condensed) phase, and 2 to the higher temperature (uncondensed or normal) phase. In the uncondensed phase, a quadratic form for the energy, which follows from a low temperature Fermi gas, is used approximation of a normal Fermi liquid,

$$
\mathrm{E}_{2}(T)=a_{2}+k_{2} T^{2}
$$

where $a_{2}$ and $k_{2}$ are constants. From the relations for the specific heat terms of $\mathrm{E}$ and the entropy S,

$$
\mathrm{C}_{V}=\frac{\partial \mathrm{E}}{\partial T}=T \frac{\partial \mathrm{S}}{\partial T}
$$

one obtains the entropy in the uncondensed phase,

$$
\mathrm{S}_{2}(T)=C_{2}+2 k_{2} T
$$

where $C_{2}$ is an unknown integration constant which later cancels out of the calculation. 
From eqs. (5) and (17) the free energy in the uncondensed phase is given by

$$
\mathrm{F}_{2}(T)=a_{2}-C_{2} T-k_{2} T^{2}
$$

The free energy in the condensed phase is obtained from the Landau expansion [4] for the free energy in terms of an order parameter $\eta$ which goes to zero at the transition to the uncondensed phase. This order parameter vanishes at a critical temperature $T_{c}$. The free energy expansion to order $\eta^{4}$ is

$$
\mathrm{F}_{1}(T, \eta)=\mathrm{F}_{2}+A \eta^{2}+B \eta^{4}
$$

Here $A$ and $B$ are functions of temperature and it is assumed that the states with $\eta=0$ and $\eta \neq 0$ are of different symmetry. In this case it can be shown the linear term in $\eta$ must be set equal to zero and if the critical point is also a stable point, e.g. if $\mathrm{F}_{1}$ as a function of $\eta$ is a minimum at $\eta=0$, then the third order term in $\eta$ should be zero and at the critical point [4]

$$
A=0 \quad B>0
$$

The order parameter is determined by requiring the condensed phase to be stable below $T_{c}$ (i.e. $F_{1}$ should be minimized w.r.t. $\eta$ ). This leads to

$$
\mathrm{F}_{1}=\mathrm{F}_{2}-\frac{A^{2}}{4 B}
$$

Furthermore, since $A$ is of opposite sign in the condensed and uncondensed phases, while $B$ is strictly positive [4], the lowest order expansion of $A$ in $T-T_{c}$ can be parametrized as

$$
A(T)=a\left(T-T_{c}\right) 2 \sqrt{B\left(T_{c}\right)} .
$$

Note especially that $a>0$ is an essential requirement following from the phase diagram [4]. Substituting for $A(T)$, the free energy per nucleon near $T_{c}$ is given by

$$
\mathrm{F}_{1}(T)=\left(a_{2}-a^{2} T_{c}^{2}\right)+\left(2 a^{2} T_{c}-C_{2}\right) T-\left(k_{2}+a^{2}\right) T^{2},
$$

where $F_{2}$ is taken from eq. (8).

From eq. (121), the energy in the condensed is easily determined to be phase near $T_{c}$,

$$
\begin{aligned}
\mathrm{E}_{1}(T) & =\left(a_{2}-a^{2} T_{c}^{2}\right)+\left(a^{2}+k_{2}\right) T^{2} \\
& =a_{1}+k_{1} T^{2} .
\end{aligned}
$$


Comparing this to the uncondensed phase (eq. (15)) we note that the $T$ dependence is also quadratic, but has a larger coefficient. Thus the specific heat is discontinuous across the phase transition, and is necessarily larger $\left(k_{1}>k_{2}\right)$ in the condensed phase.

We now apply our theoretical results to the following even-even nuclei, namely, ${ }^{20} \mathrm{Ne},{ }^{48} \mathrm{Ca}$, ${ }^{88} \mathrm{Sr}$ and ${ }^{208} \mathrm{~Pb}$. These nuclei span a large portion of the mass spectrum and have a reasonably well determined energy spectrum both in terms of their energies and the corresponding angular momentum assignments. For each of these nuclei, the partition function (see eq. (1)) contains a discrete as well as a continuum contribution.

The discrete portion of the partition function is determined empirically from the measured energy levels in each nuclei[17]. At lower excitation energies the angular momemtum assignment to each state in the spectrum is generally unique. As the energy rises, predominantly near the onset of the continuum, states occur which have an uncertainty in the angular momentum assignment. In such cases, we have taken the lowest suggested value of the angular momentum. The continuum contribution to the partition function is given by equation (2) and the value of the parameters used for the even-even-nuclei considered are given in Table 1.

The energy at which the continuum is to be attached to the discrete portion of the energy spectrum is determined via the prescription of Gilbert and Cameron[17]. This matching point was ascertained graphically by first plotting the energy as a function of the number of levels. There are two parts in each such graph, viz, the curve corresponding to the lowenergy bound states, and the curve corresponding to the high energy continuum states. The point of tangency (i.e. where the slope is the same in both curves) was then determined and selected as the matching point. They found that this could be parameterized by the following

$$
E_{c}=U_{x}+E_{p}
$$

In the above equation, $E_{c}$ is the energy at the matching point and $E_{p}$ is the pairing energy associated with the nucleus under consideration. $U_{x}$ is an additional energy term which is found graphically from the tangency point (minus the pairing energy) as a function of mass number (see Gilbert and Cameron[17]). From the graphical results one can see that there is an upper and lower limit to $U_{x}$, that the curves are hyperbolas, and that the overall behaviour is a decrease in $U_{x}$ with increasing $A$. The range for $U_{x}$ is listed below. On average, 
TABLE I: Parameters used for nuclei

\begin{tabular}{|c|c|c|c|c|c|c|}
\hline Nuclei & $P(Z)(\mathrm{MeV})$ & $P(N)(\mathrm{MeV})$ & $S(Z)(\mathrm{MeV})$ & $S(N)(\mathrm{MeV})$ & $a\left(\mathrm{MeV}^{-1}\right)$ & $E_{c}(\mathrm{MeV})$ \\
\hline${ }^{208} \mathrm{~Pb}$ & 0.83 & 0.38 & -8.86 & -3.16 & 6.61 & 6.25 \\
${ }^{88} \mathrm{Sr}$ & 1.24 & 0.93 & -16.41 & 12.88 & 9.65 & 7.14 \\
${ }^{48} \mathrm{Ca}$ & 1.83 & 1.3 & -12.07 & 12.13 & 6.80 & 10 \\
${ }^{20} \mathrm{Ne}$ & 2.5 & 2.5 & -0.811 & 5.633 & 4.58 & 17.7 \\
\hline
\end{tabular}

$U_{x}=2.5+\frac{150}{A}$,

the upper limit is,$U_{x}=2.7+\frac{200}{A}$,

and the lower limit is, $U_{x}=2.1+\frac{120}{A}$.

We have used the upper limit in all our calculations, except for ${ }^{208} \mathrm{~Pb}$ as it produced the best fit to a straight line in the uncondensed portion of the specific heat (see figures 1-4).

For ${ }^{208} \mathrm{~Pb}$ it is extremely difficult to determine the density of states. It has been pointed out by Gilbert and Cameron that not all nuclei near closed shells could be fitted (i.e. finding the tangency point) in the aforementioned manner. For those that could be fitted, a value for $U_{x}$ was found that was much larger than the one predicted. ${ }^{208} \mathrm{~Pb}$ is such a case. One can determine $U_{x}$ from the graphical results given in Gilbert and Cameron (this value is roughly $4.8 \mathrm{MeV}$ ). Adding the pairing energy to this yields a matching point value of roughly 6.5 $\mathrm{MeV}$. We used a slightly lower value. The parameters used in the determination of the partition function as a function of temperature for the nuclei under consideration are given in Table 1.

For all four nuclei (see Figures 1-4), a well defined peak is observed in the specific heat ( equation(41) ) as a function of temperature and the Landau-Ginzburg fits to the specific heat are consistent with that of a remnant of a second order phase transition. The discontinuity in the specific heat expected in an infinite system appears to be smoothed over at the critical temperature due to finite size effects. Furthermore, not unexpectedly at higher tempertures above the critical temperature, the specifc heat is linear as a function of temperature.

Varying the values of the matching points for the four nuclei considered gives rise no significant change in quantities like the critical temperature (variation of $0.01 \mathrm{MeV}$ ), or to the slope of the straight line fit (in the uncondensed phase) to the specific heat at higher 


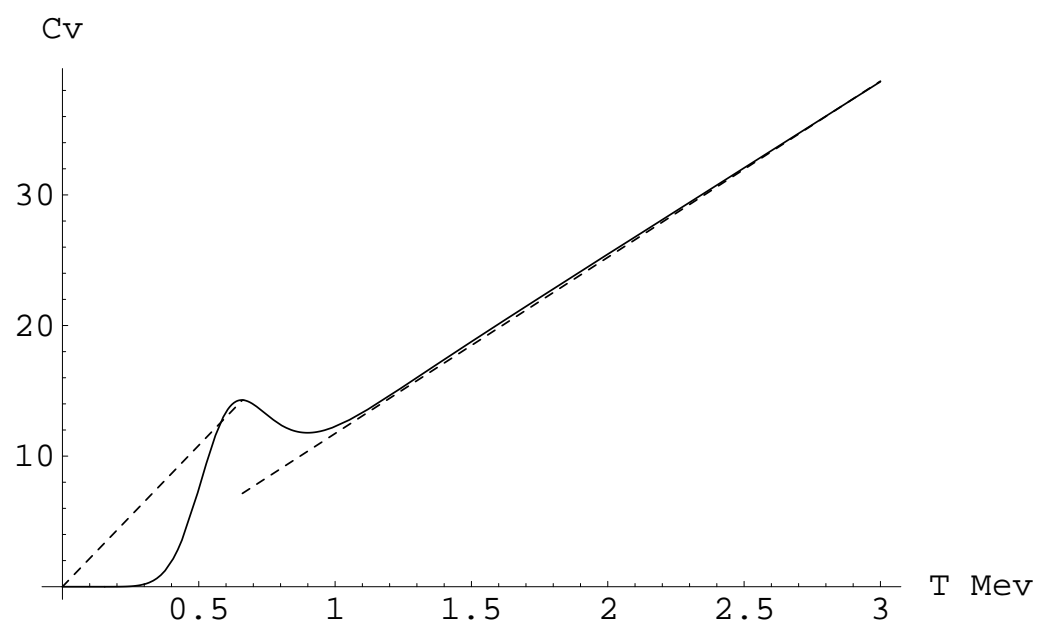

FIG. 1: Specific heat of ${ }^{208} \mathrm{~Pb}$ as a function of temperature for the parameters given in Table 1 . The dashed curve is the Landau-Ginzburg fit to the specific heat, whereas the solid curve is the specific heat determined from the experimental data. . $k 1=10.83$ and $k 2=6.75$

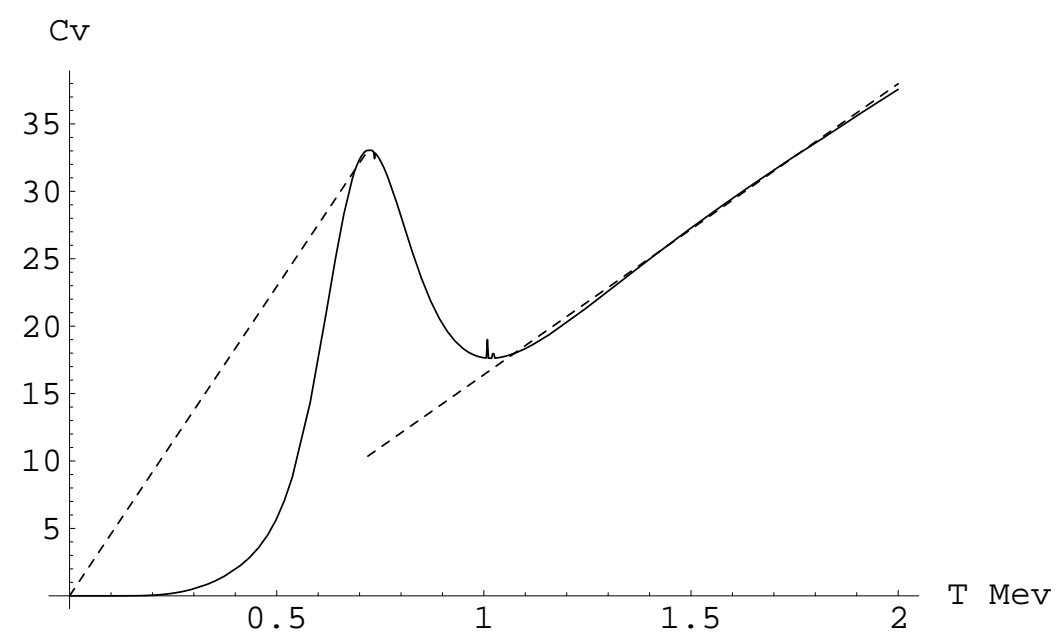

FIG. 2: Specific heat of ${ }^{88} \mathrm{Sr}$ as a function of temperature for the parameters given in Table 1. The dashed curve is the Landau-Ginzburg fit to the specific heat, whereas the solid curve is the specific heat determined from the experimetnal data. . $k 1=22.94$ and $k 2=10.79$

temperatures. The numerical value of the observed peak in the specific heat for each nucleus does vary slightly.

In an infinite system for a pairing phase transition, the discontinuity in the specific heat 


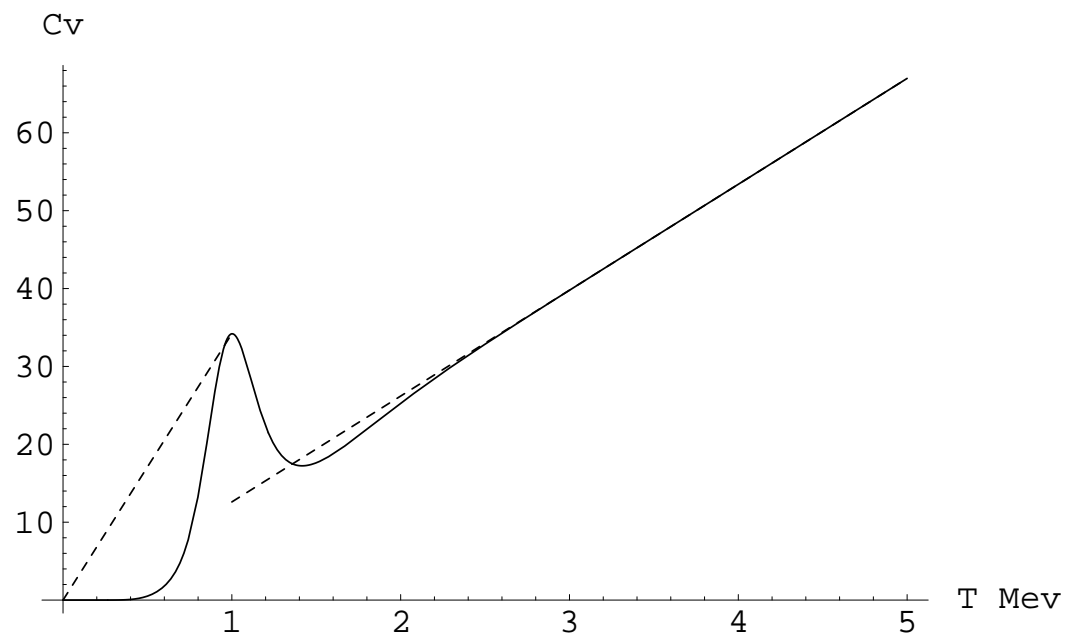

FIG. 3: Specific heat of ${ }^{48} \mathrm{Ca}$ as a function of temperature for the parameters given in Table 1. The dashed curve is the Landau-Ginzburg fit to the specific heat, whereas the solid curve is the specific heat determined from the experimental data. . $k 1=17.10$ and $k 2=6.80$

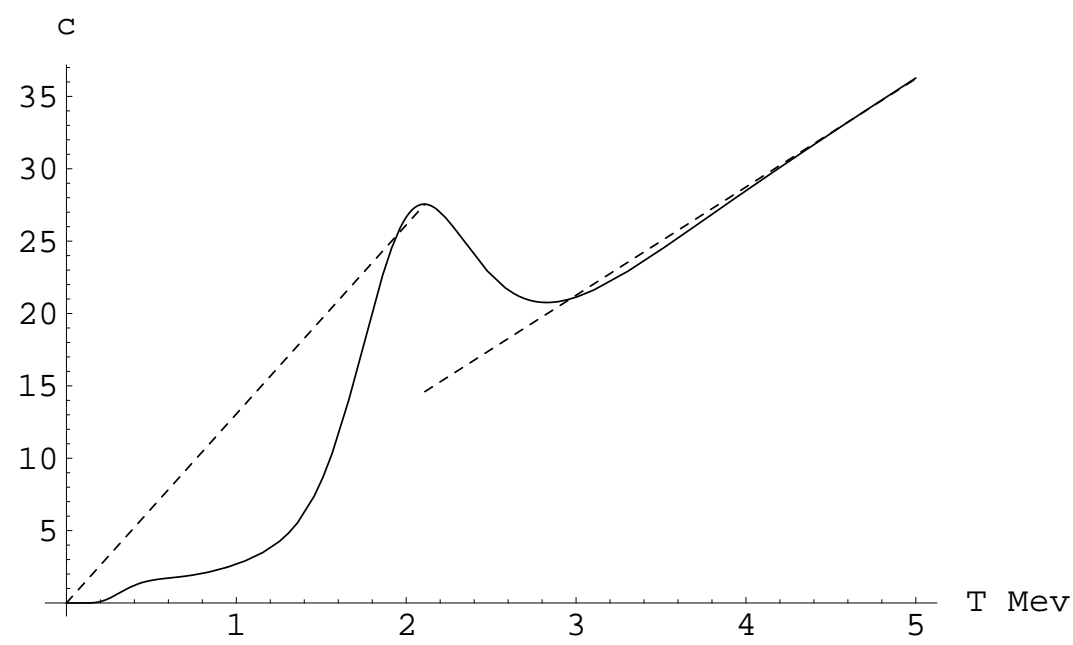

FIG. 4: Specific heat of ${ }^{20} \mathrm{Ne}$ as a function of temperature for the parameters given in Table 1. The dashed curve is the Landau-Ginzburg fit to the specific heat, whereas the solid curve is the specific heat determined from the experimental data. . $k 1=6.53$ and $k 2=3.74$

at $T_{c}$ can easily be analytically determined [4] and is given by

$$
\begin{aligned}
\frac{\mathrm{C}_{s}\left(T_{c}\right)-\mathrm{C}_{n}\left(T_{c}\right)}{\mathrm{C}_{n}\left(T_{c}\right)} & =\frac{V \frac{4 m p_{f} T_{c}}{7 \zeta(3) \hbar^{3}}}{V \frac{m p_{f} T_{c}}{3 \hbar^{3}}} \\
& =\frac{12}{7 \zeta(3)}
\end{aligned}
$$


and one can define

$$
\begin{aligned}
\Delta_{L} & =\mathrm{C}_{s}\left(T_{c}\right)-\mathrm{C}_{n}\left(T_{c}\right) \\
& =1.43 \mathrm{C}_{n}\left(T_{c}\right)
\end{aligned}
$$

Clearly in order to calculate the remnant of this discontinuity in $\mathrm{C}$ for a nucleus, this result should be corrected for finite size effects. A simple way of modeling this is to assume that the equilibrated system is contained in a finite sized volume. The sum over the momentum states in quantities like the partition function can be approximated then by an integral over the density of states in the form [32, 33]

$$
\Sigma_{p} \rightarrow \int \frac{V}{2 \pi^{2} \hbar^{3}} p^{2} d p \pm \frac{S}{8 \pi \hbar^{2}} p d p+\frac{L}{8 \pi \hbar} d p
$$

where $S$ and $L$ are the surface area and linear dimension of the system of volume $V$. The \pm correspond to either choosing Dirchlet (-) or von Neumann (+) boundary conditions. In the following we shall use Dirchlet boundary conditions and neglect the contribution from the linear term.

From Liftshitz and Pitaevskii [4] it easy to see that there is no change in the numerator of equations (16 and 17) when finite size effects are included and that only the surface term must be taken into account in the calculation of $C_{n}$ in the denominator. The surface contribution to $\mathrm{C}_{n}$ is given by

$$
\mathrm{C}_{n}^{\text {surf }}=\frac{\partial \mathbf{E}_{n}^{\text {surf }}}{\partial T}
$$

where

$$
\begin{aligned}
\mathrm{E}_{n}^{\text {surf }} & =-\frac{2 S}{8 \pi \hbar^{2}} \int \frac{\frac{p^{2}}{2 m} p d p}{z^{-1} \exp ^{\frac{\beta p^{2}}{2 m}}+1} \\
& =-\frac{2 S m T^{2}}{8 \pi \hbar^{2}} \int \frac{x d x}{z^{-1} \exp ^{x}+1} \\
& =-\frac{2 S m T^{2}}{8 \pi \hbar^{2}} F_{2}(z) \\
& =-\frac{2 S m T^{2}}{8 \pi \hbar^{2}} \frac{\epsilon_{f}^{2}}{2 T^{2}}\left[1+\frac{2 \pi^{2}}{6 \frac{\epsilon_{f}^{2}}{T^{2}}} \ldots\right]
\end{aligned}
$$

where $\epsilon_{f}$ is the Fermi energy $z=e^{\beta \mu}$ is the fugacity and $F_{2}(z)$ is the well known Fermi-Dirac integral [32]. From this it follows that the surface contribution to the specific heat for finite 
nuclei is given by

$$
\mathrm{C}_{v}^{\text {surf }} \approx-\frac{V m p_{f} T}{3 \hbar^{3}}\left[\frac{S \pi \hbar}{4 V p_{f}} \ldots\right]
$$

and that

$$
\begin{aligned}
\frac{\mathrm{C}_{s}^{\text {finite }}\left(T_{c}\right)-\mathrm{C}_{n}^{\text {finite }}\left(T_{c}\right)}{\mathrm{C}_{n}^{\text {finite }}\left(T_{c}\right)} & \approx \frac{V \frac{4 m p_{f} T_{c}}{7 \zeta(3) \hbar^{3}}}{V \frac{m p_{f} T_{c}}{3 \hbar^{3}}\left[1-\frac{3 S \hbar}{2 V p_{f}}\right]} \\
& =\frac{1.43}{\left[1-\frac{S \pi \hbar}{4 V p_{f}}\right]}
\end{aligned}
$$

and in same manner one can define

$$
\begin{aligned}
\Delta_{L}^{\text {finite }} & =\mathrm{C}_{s}^{\text {finite }}\left(T_{c}\right)-\mathrm{C}_{n}^{\text {finite }}\left(T_{c}\right) \\
& =\frac{1.43}{\left[1-\frac{S \pi \hbar}{4 V p_{f}}\right]} C_{n}^{\text {finite }}\left(T_{c}\right)
\end{aligned}
$$

Here the the Fermi momentum, $p_{F}$, for a nucleus with $A$ nucleons is determined from

$$
A=\frac{8 \pi p_{f}^{3}}{3(2 \pi \hbar)^{3}}
$$

and for simplicity $V=\frac{4 \pi R^{3}}{3}$ and $S=4 \pi R^{2}$ where $R=r_{0} A^{\frac{1}{3}}$.

After the Landau-Ginburg fits to the empirical values of the specific heat have been obtained (see figures 1-4) it is easy to determine the value of the remnant of the discontinuity (hereafter we shall refer to this as the discontinuity) in the specific heat at $T_{c}$ for each of the nuclei (see TableII). As one goes to heavier nuclei $T_{c}$ falls off hyperbolically and reaches for ${ }^{208} \mathrm{~Pb}$ a value which is slightly less than that in symmetric nuclear matter [2]. If one now assumes that this discontinuity is due to the remnant of a pairing phase transition, its value with $\left(\Delta_{L}^{\text {finite }}\right)$ and without finite size corrections $\left(\Delta_{L}\right)$ can easily be obtained from from equations (31) and (20). In spite of the simplicity of the pairing calculation(the density of states and the pairing interaction are assumed to be constant) and the difficulties with the continuum contribution (for ${ }^{208} \mathrm{~Pb}$ ) the value of the discontinuity calculated with or without finite size corrections is in reasonable agreement with the empirical value for the three heavier nuclei. Note that in the heavier nuclei the finite size corrections are not large. The discrepancy in all cases is less than $\approx 40 \%$. Only in the case of ${ }^{20} \mathrm{Ne}$ is the discrepancy about $60 \%$, more than 1.5 the value for heavier nuclei. This strongly suggests the existence of a pairing transition in the heavier nuclei which is not present in ${ }^{20} N e$. In the latter case this evidence for a second order transition which probably is shape related 19$]$. 
TABLE II: The values of the discontinuity in the specific heat at $T_{c}$

\begin{tabular}{|c|c|c|c|c|}
\hline Nuclei & $\Delta$ & $\Delta_{L}$ & $\Delta_{L}^{\text {finite }}$ & $T_{c}(\mathrm{MeV})$ \\
\hline${ }^{208} \mathrm{~Pb}$ & 7.15 & 10.22 & 10.27 & 0.66 \\
${ }^{88} \mathrm{Sr}$ & 22.67 & 14.81 & 14.92 & 0.72 \\
${ }^{48} \mathrm{Ca}$ & 21.59 & 18.02 & 18.27 & 1.0 \\
${ }^{20} \mathrm{Ne}$ & 12.95 & 20.88 & 21.46 & 2.11 \\
\hline
\end{tabular}

From the spectra of four even-even nuclei ${ }^{20} \mathrm{Ne},{ }^{48} \mathrm{Ca},{ }^{88} \mathrm{Sr}$ and ${ }^{208} \mathrm{~Pb}$ we have empirically constructed in the Canonical Ensemble their partiton functions from available experimental data and determined their specific heat as a function of temperature . For each nuclei, the specific heat displays a prominent peak which may be the remnant of a phase transition. A Landau Ginzburg treatment shows unambiguously that this interpretation is not inconsistent if the the phase transition is second order. A simple pairing calculation of the magnitude of the observed discontinuity is consistent with that obtained empirically for the three heavier nuclei. This suggests that a pairing transition takes place in these nuclei. In the case of ${ }^{20} \mathrm{Ne}$ such is not the case and the transition may be shape related.

Lastly we wish to point out that the methodology that we have employed here can be used for any finite fermion system. Given the exact spectrum of the system, the Landau-Ginsburg method can be utilized to identify the existence of the remnant of a phase transition. In cases such as pairing phase transitions, simple analytical calculations can be used to identify the nature of the phase transition.

AP acknowledges support from the Argentine National Science Council. HGM acknowledges the hospitality of the Physics Department of SUNY at Buffalo where part of this work was undertaken. 
[1] N. J. Davidson, S. S. Hsaio, J. Markram, H. G. Miller, and Y. Tzeng, Phys. Lett. B 315, 12 (1993).

[2] R. A. Ritchie, H. G. Miller, and F. C. Khanna, Eur. Phys. J. A10, 97 (2001).

[3] P. A. Seeger, Nucl. Phys. 25, 1 (1961).

[4] E. Liftshitz and L. P. Pitaeevskii, Statistical Physics II (Pergamon, Oxford, 1980).

[5] M. Baldo, J. Cugnon, A. Lejeune, and U. Lombardo, Nucl. Phys. A 515, 409 (1990).

[6] M. Lacombe et al., Phys. Rev. C21, 861 (1980).

[7] V. L. Ginzburg, J. Stat. Phys 1, 3 (1969).

[8] G. Baym, C. Pethick, D. Pines, and M. Ruderman, Nature 224, 872 (1969).

[9] J. W. Clark and N. C. Chao, Lett. Nuovo Cim. 2, 185 (1969).

[10] J. W. Clark, Phys. Rev. Lett. 23, 1463 (1969).

[11] E. Ostgaard, Nucl. Phys. A154, 202 (1970).

[12] C. H. Yang and J. W. Clark, Nucl. Phys. A174, 49 (1971).

[13] N. Chao, J. W. Clark, and C. H. Yang, Nucl. Phys. A179, 320 (1972).

[14] T. Tatsuka, Prog. Theor. Phys 48, 1517 (1972).

[15] L. Amundsen and E. Ostgaard, Nucl. Phys. A437, 487 (1985).

[16] V. J. Emery and A. M. Sessler, Phys. Rev. 114, 1377 (1960).

[17] A. Gilbert and A. G. W. Cameron, Can. J. Phys. 43, 1446 (1965).

[18] H. G. Miller, B. J. Cole, and R. M. Quick, Phys. Rev. Lett. 63, 1922 (1989).

[19] S. Levit and Y. Alhassid, Nucl. Phys. A 413, 439 (1984).

[20] H. G. Miller, R. M. Quick, and B. J. Cole, Phys. Rev. C 39, 1599 (1989).

[21] J. Dukelsky, A. Poves, and J. Retamosa, Phys. Rev. C 44, 2872 (1991).

[22] R. Rossignoli, R. M. Quick, and H. G. Miller, Phys. Lett. B 277, 18 (1992).

[23] O. Civitarese and M. Schvellinger, Phys. Rev. C 49, 1976 (1994).

[24] G. D. Yen and H. G. Miller, Mod. Phys. Lett. A 7, 1503 (1992).

[25] G. D. Yen and H. G. Miller, Phys. Rev. C 50, 807 (1994).

[26] B. J. Cole, H. G. Miller, and R. M. Quick, Mod. Phys Lett A13, 2705 (1998).

[27] D. H. Feng, R. Gilmore, and L. M. Narducci, Phys. Rev. C 19, 1119 (1979).

[28] R. Rossignoli and A. Plastino, Phys. Rev. C 32, 1041 (1985). 
[29] E. D. Davis and H. G. Miller, Phys. Lett. B 196, 277 (1987).

[30] T.Duget and P.Bonche, nucl-th/0411023 (2004).

[31] D. J. . Dean and M. Hjorth-Jensen, Rev. Mod.Phys. 75, 607 (2003).

[32] R. K. Pathria, Statistical Mechanics (Pergamon Press, Oxford, 1984).

[33] F. Solms, H. G. Miller, and A. Plastino, Phys. Lett. A 157, 286 (1991). 